\title{
DESARROLLO DEL CONOCIMIENTO FONOLÓGICO EN NIÑOS DE 5 AÑOS DE ESCUELAS PÚBLICAS DEL SECTOR RURAL EN COLOMBIA
}

\author{
Silvia Liliana Florez Trochez \\ Universidad Metropolitana (UM) (Colombia)
}

\begin{abstract}
Resumen. El presente artículo muestra el resultado del trabajo de 10 profesoras licenciadas en pedagogía infantil, quienes implementaron un modelo de intervención educativo en niños de transición de 10 escuelas públicas, ubicadas en el departamento del Atlántico (Colombia); para evaluar su contribución, en el fortalecimiento de las habilidades de conciencia fonológica. La población fue seleccionada por muestreo no probabilístico; el enfoque de la investigación fue mixto, diseño exploratorio secuencial, enmarcado en la investigación acción. La muestra estuvo compuesta por 116 niños para el grupo cuasiexperimental y 40 niños para el grupo control quienes fueron sometidos a evaluación pretest y postest a través de la Prueba de Conocimiento Fonológico -PECO-. Para el análisis estadístico comparativo de los datos cuantitativos, se utilizó el paquete estadístico Statgraphics, versión 161.15 y para los datos cualitativos, se emplearon categorías de análisis identificadas mediante el uso del software NVIVO; los datos complementarios se recogieron a través de técnicas como encuesta, entrevista semiestructurada, observación directa y revisión documental. Finalizada la implementación, todos los cursos intervenidos tuvieron avances significativos en las habilidades de conciencia fonológica (niveles silábico y fonémico) y en las tareas de identificación, omisión y adición, lo que permite afirmar que las actividades metalingüísticas, relacionadas con el conocimiento fonológico, pueden llegar a ser parte de cualquier proyecto de aula, trabajarse de manera transversal en todo programa de intervención pedagógica y con cualquier contenido curricular, como andamiaje para el aprendizaje del código lector, especialmente en aquellos casos donde los niños cursan un solo grado de preescolar.
\end{abstract}

Palabras clave: Estimulación, conciencia fonológica, lectoescritura.

\section{PHONOLOGICAL KNOWLEDGE DEVELOPMENT OF 5-YEAR OLD CHILDREN FROM PUBLIC SCHOOLS IN RURAL AREAS IN COLOMBIA}

\begin{abstract}
Summary. The current article shows the most relevant results of the work done by 10 licensed teachers in children's pedagogy, who implemented an educational intervention model in kinder children from 10 public schools, located in the department of Atlántico (Colombia); to evaluate their contribution, in the strengthening of phonological awareness skills. The population was selected by non-probabilistic sampling. The research focused on using a sequential exploratory design mixed method design, framed in action research. The sample consisted of 116 children for intervention group and 40 children for control group who were submitted to pre and post test evaluation through the Phonological Knowledge Test (PECO). For comparative statistical analysis of quantitative data, a statistical package Statgraphics version 161.15 was used and for qualitative data, we used categories of analysis identified by using NVIVO Software; the complementary data were collected through techniques such as survey, semistructured interview, direct observation and document review. After finalizing the implementation, all the grades(classrooms) intervened had significant advances in phonological awareness skills (phonemic levels and syllabic) and in identification, omission and addition tasks, which allows to affirm that metalinguistic activities can become part of any Classroom project and be implemented throughout(transversally) any program of pedagogical intervention and curricular content, as an aid for learning the reading code, specially in those cases where children just undergo one preschool grade.
\end{abstract}


Keywords: Stimulation, phonological awareness, reading and writing skills.

\title{
DESENVOLVIMENTO DO CONHECIMENTO FONOLOGICO EM CRIANÇAS DE 5 ANOS DAS ESCOLAS PUBLICAS NO SETOR RURAL DA COLOMBIA
}

\begin{abstract}
Resumo. Este artigo evidencia os resultados do trabalho de 10 professores da área de pedagogia infantil, que implementaram um modelo de intervenção educacional em crianças da transição de 10 escolas públicas, localizadas no estado do Atlântico (Colômbia); para avaliar a contribuição deles, no fortalecimento das habilidades da consciência fonológica. A população foi selecionada por amostragem não probabilística; $\mathrm{O}$ foco da pesquisa foi misto (qualitativo/quantitativo), com o desenho exploratório sequencial, enquadrado em pesquisa de ação colaborativa. A amostra consistiu-se de 119 crianças para o grupo intervenção e 40 crianças para o grupo controle submetidas à avaliação pré e pós-teste através do Teste de Conhecimento Fonológico (PECO). Para a análise estatística comparativa dos dados quantitativos, utilizou-se o software estatístico Statgraphics versão 161.15 e, para os dados qualitativos, utilizamos as categorias de análise identificadas usando o software NVIVO; os dados complementares foram coletados através de técnicas como inquérito, entrevista semiestruturada, observação direta e revisão documental. No final da implementação, todos os cursos interpostos apresentaram avanços significativos nas habilidades de conscientização fonológica (níveis silábicos e fonêmicos) e nas tarefas de identificação, omissão e adição, o que permite afirmar que as atividades metalinguísticas relacionadas ao conhecimento fonológico podem atingir faça parte de qualquer projeto de sala de aula e trabalhe transversalmente em qualquer programa de intervenção educacional e qualquer conteúdo curricular, mesmo assim ele funciona como um aspeto muito importante no aprendizado da leitura, especialmente nos casos em que as crianças cursam uma única grado escolar.
\end{abstract}

Palavras-chave: Estimulação, consciência fonológica, alfabetização.

\section{Introducción}

El presente artículo, da cuenta del resultado de la última fase, puesta en marcha, de una investigación-acción, realizada en escuelas públicas, en un contexto rural, del departamento del Atlántico en Colombia; no obstante, se considera pertinente describir las dos fases precedentes, para una visión general del proceso de investigación.

En primera instancia, la fase de sensibilización, donde las docentes participantes hicieron su primer acercamiento conceptual al tema de investigación, así como al conocimiento de experiencias exitosas relacionadas con el conocimiento fonológico y el dominio de habilidades para el alfabetismo. En segundo lugar, la de construcción acción-colaboración, donde las maestras, sirvieron de referente a la investigadora para determinar en gran parte, los fundamentos pedagógicos y didácticos del modelo de intervención. Durante este periodo, las profesoras validaron las actividades diseñadas para los niños e hicieron propuestas relacionadas con las estrategias y tipo de material a utilizar.

Durante esta fase, puesta en marcha, las docentes participantes implementaron el modelo de intervención durante un periodo de 6 meses, con su grupo de prelectores y en sus respectivas instituciones. Previo al inicio del trabajo, los niños fueron evaluados, a fin de establecer una línea base de desempeño en conocimiento fonológico y a manera 
de referencia, para la valoración pretest y comparación de los resultados obtenidos al final del periodo de implementación.

Así mismo, se presentan los principales referentes teóricos, en los cuales se basó la investigación, los antecedentes relacionados con los objetivos y problema de investigación, el método con sus respectivas técnicas e instrumentos, la discusión y conclusiones.

\section{Revisión de la literatura}

Los predictores de la lectura hacen referencia a aquellas variables relacionadas con el niño o su entorno que están vinculadas de manera directa con su adquisición; una habilidad cognitiva considerada como precursora importante es el procesamiento fonológico, entendido como destreza metalingüística que permite al usuario de una lengua reflexionar sobre los elementos fonológicos estructurales del habla y manipularlos; es decir, operar explícitamente con los segmentos de las palabras: sílabas y fonemas. (Mejía y Eslava 2008).

En el alfabeto español existen 27 letras, 29 grafemas y 25 fonemas $^{1}$ (por ejemplo: la palabra cuchillo tiene 6 fonemas $/ \mathrm{c} /, / \mathrm{u} /, / \mathrm{ch} /, / \mathrm{i} /, / 1 \mathrm{l} / \mathrm{y} / \mathrm{o} /{ }^{2}$, ocho letras c-u-ch-i-1-1-o y seis grafemas c-u-ch-i-1l-o). En el caso de su lectura, el idioma hispano es transparente, ya que existe una correspondencia biunívoca en el sentido de que, a cada grafema, le corresponde un solo fonema. En el caso de la escritura, la correspondencia varía, pues a algunos fonemas les corresponden dos o más grafemas; por ejemplo: el sonido $/ \mathrm{k} /$ se puede representar por los grafemas c y k.

Al ser el sistema de escritura, alfabético, exige el empleo de una ruta fonológica para acceder al significado, lo que implica la necesidad de que los niños aprendan la correspondencia fonema-grafema, ya que la palabra es el componente central en el proceso lector. Por tanto, su identificación es un complejo proceso de decodificación que requiere de casi todas las habilidades cognitivas del niño. Como señala Defior (2008) el lector experto es aquel que ha aprendido que las letras del alfabeto representan, en mayor o menor grado, los sonidos del habla; un conocimiento que los niños no poseen por sí mismos, sino que requieren de una intervención pedagógica para su aprendizaje.

Existen diferentes modalidades de conciencia fonológica: la conciencia silábica, relacionada con la unidad de la sílaba y la conciencia fonémica, con la unidad del fonema. La conciencia silábica implica el conocimiento de que las palabras están organizadas a partir de otras unidades articulatorias y su adquisición es temprana, ya que la sílaba es una unidad natural, carácter dado por el aspecto vocálico que le acompaña, el cual favorece la percepción y producción del habla infantil (Flórez, Restrepo y Schwanenflugel, 2009).

Por otra parte, la conciencia fonémica corresponde a la habilidad para acceder y manipular intencionalmente los fonemas de una palabra, para lo cual se requiere de una representación mental de las unidades fonológicas. Se ha señalado, que las tareas metafonológicas que miden la capacidad de realizar un análisis explícito del habla en segmentos son un buen predictor de una temprana adquisición de la lectura y de la

\footnotetext{
${ }^{1}$ Los dígrafos son grupos de letras que representan un único sonido. El sistema de representación gráfica del español cuenta con 5 dígrafos: ch, ll, rr, qu y gu (estas 2 ultimas como variantes posicionales antes de [i] y [e].

${ }^{2}$ Por la naturaleza de este trabajo no se hace transcripción según alfabetos fonéticos-fonológicos.
} 
escritura. Lo anterior se debe a que un correcto manejo de estos segmentos permite el aprendizaje adecuado de la asociación grafema-fonema.

Las investigaciones han demostrado que la conciencia fonológica tiene un efecto potenciador sobre el aprendizaje del código lector, que se puede dar gracias a la intervención oportuna y adecuada del maestro (Defior, 2008; Defior y Serrano, 2011; Flórez, et al. 2009; Porta, 2012). Por consiguiente, es a partir del conocimiento, la formación y la práctica que éste desarrolla, que puede propiciar de forma sistemática, la estimulación necesaria y acorde a la madurez del niño. Si bien, la relación entre estas dos variables ha sido ampliamente estudiada, parece no estar explicita en los currículos de formación de los futuros licenciados en pedagogía infantil, ni tampoco en la normatividad vigente de muchos países, como Colombia (Viecco y Rangel, 2012; Andrade, Peña, Renowitzky y Rincón, 2014).

Rodríguez, Cruz y Portilla (2010) refieren que la conciencia fonológica no constituye una entidad homogénea; considera diferentes niveles propuestos a través de dos interpretaciones: la primera sugiere que los niveles de conciencia fonológica se establecen de acuerdo con la dificultad de las tareas, la cual puede variar dependiendo de las demandas lingüísticas, analíticas y de memoria que requieran. En este sentido, se distingue entre tareas de clasificación (las que tienen que ver con la identificación de un sonido inicial en varias palabras: /a/ en [abeja], [avión], [anillo] y tareas de segmentación (aquellas que requieren la producción o manipulación de elementos aislados: [ma]-[le]-[ta]), atribuyendo una mayor facilidad para las tareas de clasificación. La segunda interpretación, está relacionada a la conciencia de diferentes unidades lingüísticas, lo que permite que se hable de diferentes niveles en función de la unidad lingüística, objeto de reflexión y manipulación por parte del niño, ya sea palabra, sílaba o fonema; unidades que los niños aprenden a diferenciar en los ejercicios de aprestamiento, previos a la enseñanza del código lector.

\section{Problema y objetivos de investigación}

Según la Ley General de Educación Colombiana, expedida por el Ministerio de Educación Nacional (1991) la enseñanza formal de la lectura, al menos las vocales y primeras consonantes, se inicia a partir del grado cero o transición, para lo cual el niño debe contar con 5 años cumplidos. Este único grado de preescolar está estipulado para la educación pública, mientras que para la privada se inicia a los 2 años con el nivel de maternal, lo que posibilita, por un periodo aproximado de 3 años, el desarrollo de prerrequisitos motores, sensoperceptivos y lingüísticos necesarios para el proceso lecto escritor. De entrada, se puede apreciar una condición de vulnerabilidad relacionada con la desigualdad educativa para la población infantil que, por los bajos recursos económicos de sus familias o su ubicación geográfica, solo puede acceder a la educación ofertada por el estado. Si bien, el gobierno colombiano ha tratado de subsanar esta necesidad a través de los Centros de Desarrollo Infantil -CDIs- atendidos por madres cabeza de hogar, su nivel de formación dista del que puede alcanzar un profesional, licenciado en Pedagogía Infantil. Esta condición resulta preocupante, ya que bien sea desde la perspectiva de la educación pública o privada, en el proceso del desarrollo fonológico, ocurre la intervención pedagógica de aproximación a la lengua escrita; por lo cual, en la etapa preescolar, es la acción mediadora del educador la que facilita la transformación en las estructuras cognitivas de los niños involucradas en este proceso.

Por último, autores como Flórez (2009), Caldera, Escalante y Terán (2010) han demostrado que factores como el pobre conocimiento que tienen los docentes sobre el 
desarrollo fonológico y las dificultades fonológicas que pueden llegar a presentar los niños a su cargo, les impide realizar propuestas de trabajo pertinentes para su estimulación y realizar remisiones oportunas, en caso de requerirse; así mismo, que para la adecuada implementación de acciones tendientes a desarrollar habilidades de conciencia fonológica a través de tareas, y su ejecución en el plan de trabajo para cada nivel de la educación inicial, se requiere una formación previa y formal del docente.

A partir de las consideraciones presentadas, los interrogantes que esta investigación pretendió responder fueron los siguientes:

- ¿En qué medida la puesta en marcha de un modelo de intervención pedagógica estimula las habilidades de conciencia fonológica en niños pertenecientes al curso de transición en escuelas publicas?

- ¿Cuáles son los fundamentos psicopedagógicos y lingüísticos en los que se apoya el modelo de intervención, para la estimulación de las habilidades de conciencia fonológica por parte del profesorado del grado Transición?

- ¿Cómo diseñar colaborativamente un modelo de intervención que promueva el desarrollo de habilidades de conciencia fonológica, a través de actividades contextualizadas a la realidad social y educativa de los niños pertenecientes al curso de transición en escuelas publicas?

Para dar respuesta a este problema, se propuso como objetivo general:

- Evaluar la contribución del modelo de intervención para la estimulación de habilidades de conciencia fonológica en niños de transición pertenecientes a escuelas públicas.

Y como objetivos específicos:

- Determinar los fundamentos psicopedagógicos y lingüísticos de un modelo de intervención para el desarrollo de las habilidades de conciencia fonológica.

- Diseñar colaborativamente un modelo de intervención que promueva el desarrollo de habilidades de conciencia fonológica a traves de actividades que respondan a las necesidades educativas y sociales de los niños de transición, perteneicente a escuelas publicas.

\section{Investigaciones precedentes}

Si bien, los resultados de búsqueda de los precedentes afines con el tema de investigación fueron organizados en 5 categorías para su estudio, se presentan los concernientes a los objetivos relacionados con los efectos de programas sobre el desarrollo de la conciencia fonológica, la formación y prácticas docentes. Desde las perspectivas de los autores, se ubicaron investigaciones desde el 2008 hasta el 2014.

Los hallazgos relacionados con la primera categoría, dieron cuenta del efecto positivo de programas de conciencia fonológica durante la educación inicial y primeros años de la básica primaria, los cuales fueron aplicados en grupos control y de intervención constituidos por niños con y sin riesgo de problemas de aprendizaje; las propuestas de intervención variaron en la cantidad de segmentos sonoros (palabras, silabas y fonemas) y las habilidades consideradas para la estimulación (segmentación, adición, eliminación, aliteración y sustitución); además se encontró diferencia en la modalidad de trabajo evidenciada en la didáctica y material de apoyo sugerido, así como la variación en el periodo de tiempo en el cual estuvieron expuestos los niños a 
los programas (entre 2 meses y 2 años), el número se sesiones semanales realizadas ( entre 2 y 5), el tiempo de duración de cada actividad programada (de 20 a 60 minutos) y las tipologías de los grupos intervenidos, los cuales estuvieron constituidos por población preescolar y escolar (primero y segundo grado de primaria) pertenecientes a su vez a los sectores geográficos rural y urbano, instituciones educativas públicas y privadas, y finalmente, los estratos socioeconómicos a los cuales partencia la población, en este caso vulnerable y favorecida (González, López, Vilar y Rodríguez, 2013; Maldonado y Guerrero, 2009; Porta, 2012; Velarde, Canales, Meléndez y Lingán, 2011).

También se pudo demostrar, la incidencia de la estimulación de las habilidades de conciencia fonológica sobre diferentes variables como la velocidad de denominación y la lectura de pseudopalabras; un mejor rendimiento en el grupo de intervención respecto al grupo control; diferencias en cuanto a los sujetos responsables de implementar los programas siendo en la mayoría de los casos, los mismos investigadores y en menor proporción, los docentes (Acosta, Moreno y Axpe, 2011; Arancibia, Bizama y Sáez, 2014; Ghiglion, Arán y Apaz, 2011; González, Martín y Delgado, 2011).

Presentada la relación de las investigaciones precedentes, relacionadas con la implementación de programas en conocimiento fonológico se pasa a mostrar, aunque en menor proporción, las concernientes a la participación de docentes en programas de estimulación en espacios de aula y en población preescolar y escolar. En los resultados se resalta el papel del maestro, al incluir en el desarrollo de los contenidos curriculares, actividades para diferentes segmentos sonoros; favoreciendo a corto y medio plazo, el rendimiento de los niños en el aprendizaje del código lector. Así mismo, la importancia de involucrar a los padres de familia en las estrategias de alfabetismo Flórez, et al. (2009); la diversificación y frecuencia de las actividades (Auseche, 2009).

Se destaca también, el resultado de los procesos reflexivos de los maestros hacia sus prácticas pedagógicas, relacionadas con la enseñanza de la lectura y escritura que involucre contextos reales, el conocimiento de las dimensiones de los niños y sus indicadores de desarrollo; el efecto positivo, para la ganancia en la capacidad de lectura, de métodos que usen la ruta fonológica (Martins y Aparecida, 2009; Garay, 2012); las aproximaciones lúdicas que realizan las maestras de preescolar en contraposición a las de primer y segundo grado de primaria, quienes se centran en la decodificación del símbolo, la enseñanza del sonido y su correspondencia con la palabra; las experiencias propias de los docentes como lectores y la forma como enseñan a leer a sus estudiantes; es decir, la relación de vivencias positivas al leer y escribir, con la motivación e implementación de estrategias para su práctica de enseñanza (Flórez, 2009; Caldera, Escalante y Terán, 2010).

Finalizada la presentación de los antecedentes, problemática y objetivos que enmarcan el tema del presente artículo, se pasa a exponer la metodología con su respectivo diseño de investigación, población participante, técnicas e instrumentos empleados.

\section{Método}

Se recurrió a la investigación-acción como aproximación metodológica y al diseño de tipo cuasiexperimental pretest-postest con grupo control, lo que permitió dar 
respuesta a los objetivos planteados para la fase puesta en marcha, relacionados con la identificación de las concepciones y necesidades de los docentes frente al tema de conciencia fonológica; la construcción colaborativa de los fundamentos del modelo y su diseño; la aplicación y evaluación de la contribución del mismo en el desarrollo de las habilidades de conciencia fonológica. Se utilizó como técnica, la evaluación y como instrumento el formato para la evaluación de las habilidades de conciencia fonológica PECO- de Ramos y Cuadrado (2006).

Esta aproximación metodológica facilitó el conocimiento del entorno social y local de donde provenían los niños y laboraban las docentes, al tiempo que apuntó a la producción de un accionar propositivo de las maestras en la medida que, a partir de un proceso reflexivo, reconocieron las falencias respecto a su preparación en el tema del conocimiento fonológico, así como la importancia de su estimulación previa al aprendizaje del código lector. Para este diseño, la selección de la muestra fue de tipo intencional dada en función de su disponibilidad de los niños en las instituciones educativas durante el periodo escolar $(10$ meses $)$ y la intencionalidad de la investigadora, de favorecer con el programa de estimulación, a todos los niños de cada curso participante.

\section{Participantes}

Para este diseño, la selección de la muestra fue de tipo intencional o con propósito, y estuvo conformada por docenes licenciados en pedagogía infantil, nombrados por la Secretaria de Educación Departamental para el grado de transición, del sector educativo público, que laborasen con población en situación de vulnerabilidad, bajo la modalidad de participantes voluntarios los cuales fueron convocados a través de un oficio formal enviado a sus respectivos rectores por parte de la Secretaria Departamental de Educación; así mismo el grupo de estudiantes naturalmente constituidos en cada aula de clase con el siguiente criterio de exclusión: menores de 5 años, discapacidad sensorial y cognitiva.

Para el grupo de intervención se seleccionaron 10 maestras y 119 niños (Tabla 1) y para el grupo control, 4 maestras y 40 niños (Tabla 2) quienes iniciaron el grado de transición en febrero del 2016; todos los niños evaluados contaron con el consentimiento informado de sus padres, como representantes legales; en cada institución educativa, con la de sus rectores, quienes a su vez tenían la autorización del Secretario Departamental de Educación.

Tabla 1

Población grupo de intervención

\begin{tabular}{lcc}
\hline \multicolumn{1}{c}{ Municipio } & Institución Educativa & Número de Niños \\
\hline \multirow{2}{*}{ Campo de la Cruz } & Comercial la Inmaculada & 15 \\
\multirow{3}{*}{ Galapa } & Bohorquez & 13 \\
& Antonio Nariño de Paluato & 10 \\
\multirow{3}{*}{ Ponedera } & Frutos de la esperanza & 08 \\
& Martillo, primera jornada & 13 \\
& Martillo, segunda jornada & 09 \\
\hline
\end{tabular}




\begin{tabular}{lcc}
\hline \multirow{2}{*}{ Sabanalarga } & San José Aguada de pablo & 10 \\
& San Cayetano Gallego & 08 \\
Santo Tomas & Oriental sede No.5 El Carmen & 21 \\
\hline
\end{tabular}

Nota: Fuente, construcción de la autora (2018)

Tabla 2

Población grupo control

\begin{tabular}{ccc}
\hline Municipio & Institución Educativa & Número de Niños \\
\hline \multirow{3}{*}{ Puerto Colombia } & Maria Mancilla Sánchez, Transición A & 10 \\
& Maria Mancilla Sánchez, Transición B & 10 \\
& Maria Mancilla Sánchez, Transición C & 10 \\
& Maria Mancilla Sánchez, Transición D & 10 \\
\hline
\end{tabular}

Nota: Fuente, construcción de la autora (2018)

\section{Técnicas e instrumentos}

Los procedimientos utilizados para obtener la información fueron entrevistas focales y semiestructuradas, relacionadas con los conocimientos previos de las maestras sobre las habilidades de conciencia fonológica, su incidencia en el aprendizaje del código lector y las estrategias mediadoras para potenciar su desarrollo en los niños. Para la valoración de los datos obtenidos se utilizó la escala Likert y el software Nvivo; las observaciones participantes, donde se pudo evidenciar el conocimiento y apropiación de la didáctica para el desarrollo de las actividades por parte de cada docente, a la vez que se establecieron diálogos relacionados con aspectos de mejora o inquietudes sobre los registros de los avances de los niños.

También se realizaron revisiones documentales, a partir de los registros realizados por las maestras, relacionados con la planeación de las actividades semanales y las respuestas de los niños; la evaluación pretest y postest, lo que permitió establecer una línea base de desempeño de los niños antes y después de la implementación del modelo y posteriormente la respectiva comparación en la ejecución de cada tarea.

Los recursos, de corte cualitativo, utilizados para registrar la información sobre las variables estudiadas (conciencia fonológica y programa de intervención) fueron los diarios de campo, donde se registró las actividades realizadas por las maestras, el material utilizado, las facilitaciones y refuerzos positivos brindados, las dificultades en la ejecución y sugerencias a tener en cuenta en el periodo comprendido entre una visita y otra; los registros audiovisuales, relacionados con las filmaciones realizadas en cada visita de seguimiento y los textos escritos, constituidos por los parceladores, donde las maestras registraban su actividad curricular semanal.

Los instrumentos de corte cuantitativo estuvieron representados en las encuestas y protocolos de evaluación, lo que permitió un análisis de comparaciones numéricas y estadísticas y su respectiva representación en gráficas a partir de la ubicación de los niños en percentiles, de acuerdo con su nivel de desempeño en la Prueba Exploratoria para el Conocimiento Fonológico (PECO). Cada uno de los aspectos evaluados en el instrumento fueron sometidas a criterios de validación por 5 jueces expertos ( 3 a nivel nacional y 2 internacional), bajo los criterios de validez, pertinencia, claridad y completitud 
Se considera pertinente presentar un resumen de la distribución del número de elementos evaluados por la prueba, en función de los niveles (silábico y fonético), las tareas (identificación, omisión y adición), las puntuaciones directas posibles de obtener transformadas en deciles y la correspondencia con una valoración cualitativa (Tablas 3 y 4), que permitirá visualizar el avance de los niños después de la intervención.

Tabla 3

Distribución de elementos según criterios de clasificación

\begin{tabular}{cccccc}
\hline \multirow{2}{*}{ Nivel } & Posicion & \multicolumn{4}{c}{ Tareas } \\
& & Identificar & Añadir & Omitir & Total \\
\hline \multirow{2}{*}{ Silábico } & Inicial & 2 & 2 & 2 & 6 \\
& Final & 2 & 2 & 2 & 6 \\
& Medial & 1 & 1 & 1 & 3 \\
\multirow{5}{*}{ Fonémico } & Inicial & 2 & & & \\
& Final & 0 & 2 & 0 & 2 \\
& Medial & 3 & 1 & 2 & \\
& & 10 & 10 & 10 & 30 \\
\hline \multicolumn{2}{c}{ TOta: Tomado de Ramos y Cuadrado (2006). Prueba para la evaluación del conocimiento fonológico. }
\end{tabular}

En la Tabla 3 se pueden observar los niveles y tareas evaluar, así como el número de tareas consideradas para cada ítem con su respectivo puntaje; para un total de 30 .

Tabla 4

Baremos de transformación de puntuaciones directas en deciles

\begin{tabular}{cccccccc}
\hline $\begin{array}{c}\text { Valoracion } \\
\text { Cualitativa }\end{array}$ & Decil & $\begin{array}{c}\text { Identific } \\
\text { acion }\end{array}$ & Adición & Omision & $\begin{array}{c}\text { Conoc } \\
\text { Silábico }\end{array}$ & $\begin{array}{c}\text { Conoc } \\
\text { Fonémico }\end{array}$ & Total \\
\hline Muy alta & 9 & 10 & 9 & 8 & $13-14$ & 13 & $>24-$ \\
& 8 & - & 8 & 7 & - & 11 & $23-24$ \\
Alta & 7 & 9 & - & 6 & 12 & 10 & $21-22$ \\
& 6 & - & 7 & 5 & 11 & 9 & $19-20$ \\
Media & 5 & 8 & 6 & - & 10 & 8 & 18 \\
& 4 & 7 & 5 & 4 & 9 & 7 & $16-17$ \\
Baja & 3 & - & 4 & 3 & 8 & 6 & 15 \\
Muy baja & 2 & 6 & 3 & 2 & 7 & 5 & $12-14$ \\
\hline
\end{tabular}

Nota: Tomado de Ramos y Cuadrado (2006). Prueba para la evaluación del conocimiento fonológico

La Tabla 4 muestra la totalidad de puntos posibles para cada ítem evaluado y su correspondiente valoración cualitativa; la cual se constituye en un referente para que el 
docente conozca la línea base de sus estudiantes para las actividades que puede trabajar a lo largo del curso. Para el caso que compete, las 10 instituciones participantes obtuvieron un puntaje global inferior a 12 para el pretest, lo que corresponde a un nivel de desempeño muy bajo.

\section{Análisis de datos}

Para el análisis estadístico comparativo de los datos cuantitativos que arrojó del desempeño de los niños, se utilizó el software Statgraphics versión 16 1.15; programa que utiliza 3 medidas de variabilidad para mostrar el resultado: frecuencia, media y desviación estándar.

Las frecuencias, fueron establecidas a partir del agrupamiento de los datos obtenidos a partir de la valoración de los niños en 4 clases o intervalos dados por un límite inferior y otro superior; para este caso 1, 2, 3 y 4; es decir, a partir de la frecuencia en que cada puntuación de los niños se repitió, tanto por tarea (identificación, adición, omisión) como por nivel (silábico y fonémico). Los porcentajes de la última columna, frecuencia relativa, corresponden a la cantidad de niños, del total de la muestra, que se ubicaron en cada rango, siendo mayor, los que presentaron respuestas en la clase 1 ( $0-1,25$ puntos) o sea, puntajes muy bajos.

Se elaboraron tablas pretest y postest por cada segmento y tarea, tanto para el grupo de intervención como el grupo control, que incluyeron el cálculo de la media de desempeño de todos los niños y la desviación estándar, como medida que explica, la variación de las respuestas de los mismos, en relación con el desempeño promedio; las tablas de frecuencia permitieron evidenciar una clara mejora después de la intervención, tal y como se muestra en las Tablas del 5 al 10, referenciadas a manera de ejemplo, las cuales muestran los cambios de puntaje en ambos grupos (Grupo de Intervención GI y Grupo Control GC), antes y despues para las tarea de omisión, nivel silábico. En la parte inferior de cada tabla de frecuencia (Tablas 5, 6, 8 y 9), se presenta el cálculo de la media de desempeño de todos los niños, tanto en el pretest como en el postest, y la desviación estándar, como una medida que explica, la variación de las respuestas de los niños en las tareas realizadas, en relación con el desempeño promedio.

Finalmente, la verificación de la varianza o prueba de hipotésis, la cual busca hacer comparaciones entre las variaciones estadísticas de cada test o tarea realizada por los niños, a través del test de Levene (Tablas 7 y 10). Esta prueba se aplicó para determinar si la diferencia encontrada en las desviaciones estándar de las respuestas de los estudiantes, antes y después de la intervención, es estadísticamente significativa para extrapolarlas a la población.

- Hipótesis Nula: Las desviaciones de las respuestas antes y después son iguales.

- Hipótesis Alternativa: Las desviaciones de las respuestas antes y después son diferentes.

El test de Levene aplica el criterio del valor $P$, para emitir el juicio sobre la hipótesis nula. El juicio sobre la hipótesis alternativa surge dependiendo de la decisión sobre la hipótesis nula. El criterio del valor $P$ señala que si se maneja una confianza del 95\% (que es lo usualmente utilizado), entonces la hipótesis nula se acepta si el valor $P$ es mayor o igual a 0,05 (5\% de margen de error), pero si es menor de 0,05 entonces se rechazara la hipótesis nula. 
Tabla 5

Tareas omisión, nivel silábico, frecuencias pretest GI

\begin{tabular}{ccccc}
\hline Clase & $\begin{array}{c}\text { Limite } \\
\text { Inferior }\end{array}$ & $\begin{array}{c}\text { Limite } \\
\text { Superior }\end{array}$ & Frecuencia & $\begin{array}{c}\text { Frecuencia } \\
\text { Relativa }\end{array}$ \\
\hline 1 & 0 & 1,25 & 88 & $\mathbf{9 4 \%}$ \\
2 & 1,25 & 2,5 & 1 & $1 \%$ \\
3 & 2,5 & 3,75 & 2 & $2 \%$ \\
4 & 3,75 & 5,0 & 3 & $\mathbf{3 \%}$ \\
\hline
\end{tabular}

Nota: Construcción de la autora (2015)

Media $=0,234$; Desviación Estándar $=0,847$

Tabla 6

Tareas omisión nivel silábico, frecuencias postest GI

\begin{tabular}{ccccc}
\hline Clase & $\begin{array}{c}\text { Limite } \\
\text { Inferior }\end{array}$ & $\begin{array}{c}\text { Limite } \\
\text { Superior }\end{array}$ & Frecuencia & $\begin{array}{c}\text { Frecuencia } \\
\text { Relativa }\end{array}$ \\
\hline 1 & 0 & 1,25 & 55 & $\mathbf{5 8 \%}$ \\
2 & 1,25 & 2,5 & 5 & $5 \%$ \\
3 & 2,5 & 3,75 & 2 & $2 \%$ \\
4 & 3,75 & 5,0 & 32 & $\mathbf{3 5 \%}$ \\
\hline Nota: Construcción de la autora $(2017)$ & & \\
Media $=1,968 ;$ Desviación Estándar $=2,177$ &
\end{tabular}

Al comparar las tablas de frecuencia del grupo de intervención (Tablas 5 y 6), se puede evidenciar que antes de la intervención el 3\% de los niños tenían puntajes entre 4 y 5 (clase 4 pretest), pero después de la intervención el 35\% de los niños obtuvieron puntajes de 4 o 5 en la misma clase, lo que permite observar un incremento de respuestas positivas en este rango. También se puede observar cambio en la clase 1, donde disminuyó el porcentaje de niños ubicados en el rango de respuestas muy bajas, pasando del $94 \%$ al $58 \%$.

Tabla 7

Verificacion de varianza, tareas omisión nivel silábico GI

\begin{tabular}{ccc}
\hline & Prueba & Valor-P \\
\hline Levene & 86,789 & 0 \\
\hline Nota: Construccion de la autora (2017)
\end{tabular}

Dado que el test de Levene presentó un valor $P$ menor de 0,05 (Tabla 7) se puede concluir que la variabilidad en los puntajes antes y después de la intervención fue diferentes. El aumento de la variabilidad después de la intervención posiblemente se debe a que no todos los niños fueron influenciados de la misma manera, no obstante, los puntajes de desempeñó en promedio aumentaron; comprobando la hipótesis alternativa donde las desviaciones de las respuestas antes y después son diferentes. 
Tabla 8

Tareas omisión nivel silábico, frecuencias pretest $G C$

\begin{tabular}{ccccc}
\hline Clase & $\begin{array}{c}\text { Limite } \\
\text { Inferior }\end{array}$ & $\begin{array}{c}\text { Limite } \\
\text { Superior }\end{array}$ & Frecuencia & $\begin{array}{c}\text { Frecuencia } \\
\text { Relativa }\end{array}$ \\
\hline 1 & 0 & 1,25 & 30 & $\mathbf{9 7 \%}$ \\
2 & 1,25 & 2,5 & 0 & $0 \%$ \\
3 & 2,5 & 3,75 & 0 & $0 \%$ \\
4 & 3,75 & 5,0 & 1 & $\mathbf{3 \%}$ \\
\hline
\end{tabular}

Nota: Construcción de la autora (2017)

Media $=0,129 ;$ Desviación Estándar $=0,718$

Tabla 9

Tareas omisión nivel silábico, frecuencias postest GC

\begin{tabular}{ccccc}
\hline Clase & $\begin{array}{c}\text { Limite } \\
\text { Inferior }\end{array}$ & $\begin{array}{c}\text { Limite } \\
\text { Superior }\end{array}$ & Frecuencia & $\begin{array}{c}\text { Frecuencia } \\
\text { Relativa }\end{array}$ \\
\hline 1 & 0 & 1,25 & 27 & $\mathbf{8 7 \%}$ \\
2 & 1,25 & 2,5 & 0 & $0 \%$ \\
3 & 2,5 & 3,75 & 0 & $0 \%$ \\
4 & 3,75 & 5,0 & 4 & $\mathbf{1 3 \%}$ \\
\hline
\end{tabular}

Nota: Construcción de la autora (2015)

Media = 0,709; Desviación Estándar = 1,696

Por medio de la comparación de las tablas de frecuencia del grupo de control (Tablas 8 y 9), se puede evidenciar que en la medición inicial el 3\% de los niños tenían puntajes entre 4 y 5 (clase 4, pretest), pero después en la segunda medición el $13 \%$ de los niños obtuvieron puntajes de 4 o 5 (clase 4 postest). Es decir, en las tareas de omisión algunos niños avanzaron, no obstante, es poco significativo si se mira el porcentaje de la población ubicada en la primera clase, donde pasó del 97\% al 87\% . Es decir, el rango de respuestas bajas continuó siendo muy alto en el grupo control.

Tabla 10

Verificacion de varianza, tareas omisión nivel silábico GI

\begin{tabular}{ccc}
\hline & Prueba & Valor-P \\
\hline Levene & 3,0779 & 0,0844688 \\
\hline
\end{tabular}

Nota: Construcción de la autora (2017)

Dado que el test de Levene presentó un valor $P$ mayor de 0,05 se puede concluir que la variabilidad en los puntajes antes y después en el grupo control fue la misma. Es decir, la variabilidad de las respuestas frente a la media obtenida por los grupos después de la intervención fue homogénea (Tabla 10); comprobando la hipótesis nula donde las desviaciones de las respuestas antes y después son diferentes. 
Para el análisis cualitativo, se emplearon categorías de análisis identificadas médiate el uso del software NVIVO, el cual permitió organizar la información, analizar y encontrar relaciones en datos cualitativos; para este caso, fueron las consideradas en el protocolo de observación y encuesta.

\section{Resultados}

Se presentan a continuación los resultados promedio de los niños evaluados en cada institución educativa, quienes presentaron como característica común, un nivel de desempeño mayor en las tareas de identificación para los niveles silábico y fonémico; en relación con las de adición y omisión, en el pretest. Este desempeño ubicó a las 10 instituciones en el primer percentil (puntuaciones totales inferiores a 12). La ubicación en un percentil determinado es un indicador para que el maestro organice, dentro de su actividad académica, las tareas que van a ayudar a sus estudiantes a ir avanzando de una habilidad a otra.

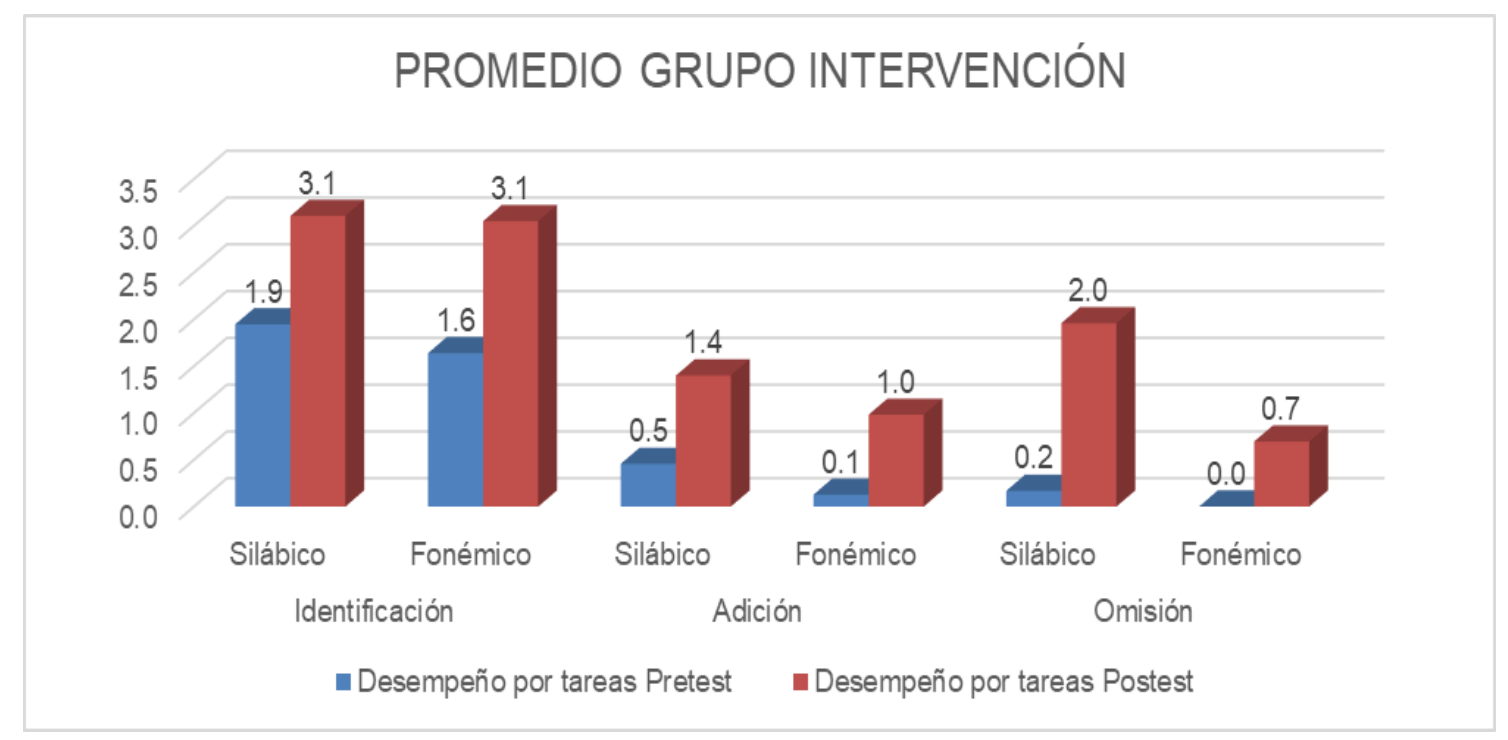

Figura 1. Desempeño general por tareas grupo de intervención pretest y postest

La Figura 1 muestra un incremento en la puntuación promedio, obtenida para todas las tareas que evaluaron el conocimiento fonológico de los niños pertenecientes a las 10 instituciones participantes a nivel silábico y fonémico en las habilidades de identificación, adición y omisión. El desempeño del grupo pasó en puntuación directa de 0.7 a 1.9 en la evaluación postest. 


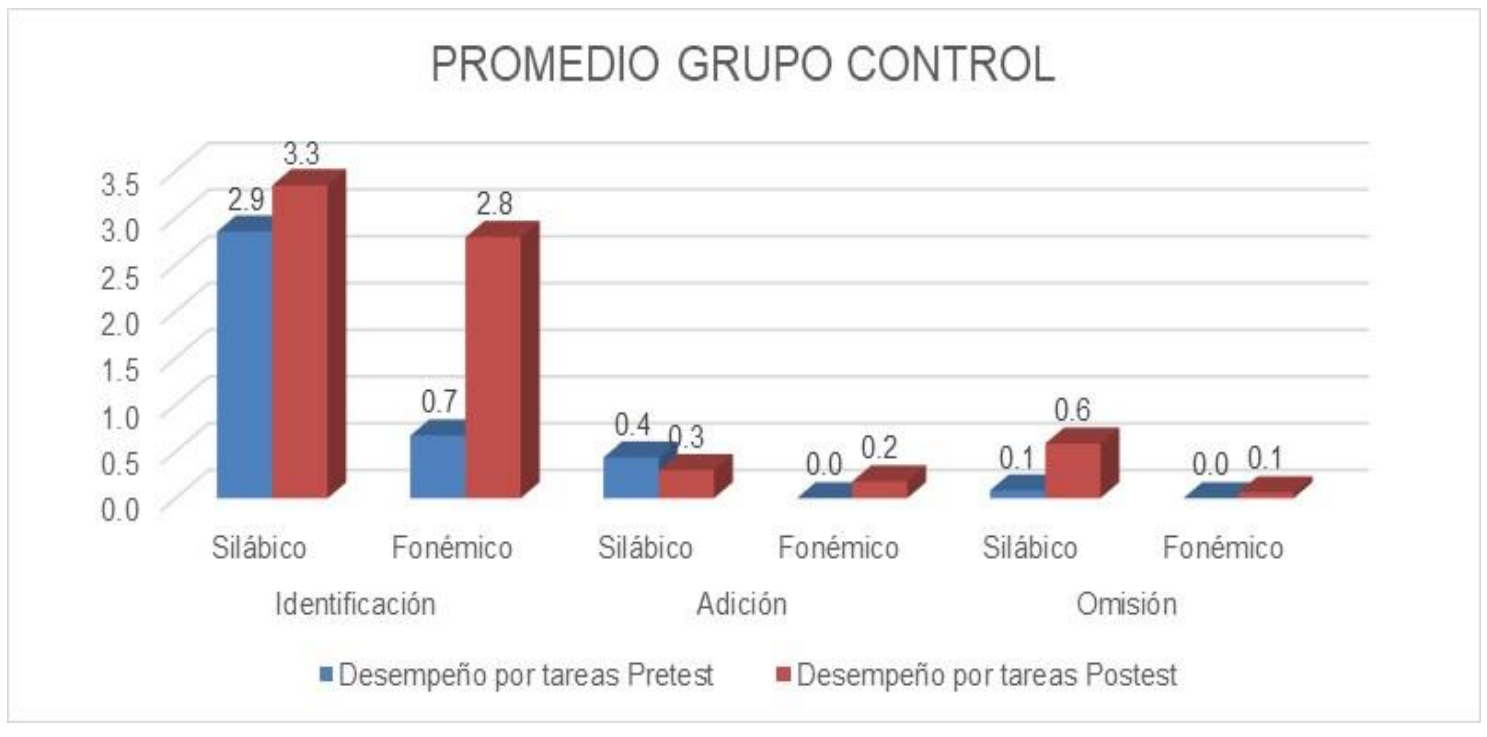

Figura 2. Desempeño general por tareas grupo control pretest y postest

En comparación con la Figura 1, en la Figura 2 se aprecia un incremento en la puntuación promedio, obtenida solo para todas las tareas de identificación, pasando de una puntuación de 2.9 a 3.3 para identificación silábica, de 0.7 a 2.8 para identificación fonémica; para las demás tareas el avance fue mínimo, lo que permite decir que, el desempeño del grupo control pasó en puntuación directa de 0.9 a 1.2 en la evaluación postest.

\section{Discusión y conclusiones}

A continuación, se exponen las principales conclusiones del estudio, de acuerdo con los objetivos planteados y algunas limitaciones que intervinieron en la diferencia de desempeño de los niños:

Con relación a la contribución del modelo de intervención, al desarrollo del conocimiento fonológico en los niños, se observó que la puntuación de desempeño en las tareas del grupo de intervención fueron significativamente más altas que la del grupo control, específicamente en las de identificación, omisión y adición, tanto a nivel silábico y fonémico; tal como sustentan los postulados de: Defior (2008), Defior et al. (2011), Flórez, et al. (2009) y Porta (2012) donde a partir del conocimiento, la formación y la práctica de los docentes, pueden propiciar de forma sistemática, la estimulación necesaria para que los niños, desarrollen el conocimiento fonológico, de acuerdo a su madurez cognitiva y lingüística; por otro lado, al ser esta habilidad metalingüística un fenómeno inestable, es posible su modificación a partir de una estimulación permanente, en la medida en que los niños sean expuestos a actividades contextualizadas durante la educación inicial, manifestada en una habilidad creciente para discriminar las diferentes unidades que componen las palabras (Villagrán, Marchena y Navarro, 2011; Fumagalli y Jaichenco, 2010).

Por lo tanto, si bien los niños intervenidos no tenían antecedentes de escolarización previa, fue posible desarrollar un programa de estimulación para el conocimiento fonológico en el grado transición, como único nivel del ciclo de 
educación inicial, a partir de una enseñanza sistemática y explicita por parte de los maestros y sin salirse de su plan de trabajo escolar; en un tiempo relativamente corto, si se compara con el empleado normalmente durante los niveles previos de prejardín y jardín; cursos que se ofertan solo en la educación privada. Ahora, si bien el nivel general de desempeño de los niños de transición de las 10 instituciones participantes fue satisfactorio, unos grupos s se destacaron más que otros; diferencia que estuvo relacionada con la oportunidad de las profesoras para poner en escena todas las consideraciones del modelo de intervención, el número de niños por aula y el índice de deserción en cada institución educativa durante el periodo que duró la implementación.

En cuanto a la determinación de los fundamentos del modelo y el diseño de las actividades, fue preponderante el aporte de las maestras desde su saber y experiencia disciplinar, ya que ayudaron a validar todas las actividades planteadas por la investigadora según habilidad y segmento sonoro, específicamente en lo relacionado con el material a utilizar, las instrucciones para los niños, la duración de las tareas y la frecuencia semanal con que se realizaron. Los resultados referentes a este objetivo permitieron demostrar que las docentes de educación inicial juegan un papel preponderante en el establecimiento de precurrentes, no solo de tipo motor y sensoperceptivo, sino lingüísticos; y de esta forma, ser de gran ayuda en el establecimiento cognitivo de habilidades que ayudan al aprendizaje de la lengua escrita.

Tal y como fue demostrado por Auseche (2009), Flórez, et al. (2009) no se puede desconocer el papel de las docentes, al incluir en el desarrollo de los contenidos curriculares, este tipo de tareas; favoreciendo a corto y medio plazo, el rendimiento de los niños en el aprendizaje del código lector. Así mismo, es importante involucrar a los padres de familia en las estrategias de alfabetismo, la diversificación y frecuencia de las actividades, como extensión al trabajo realizado en el aula.

Referente a las limitaciones que se encontraron durante el desarrollo de la investigación, se hará referencia a las relacionadas con los niños dadas por la asistencia irregular a las jornadas de clase, la fluctuación en la cantidad de estudiantes matriculados, evidenciada entre los que iniciaron con el proceso de estimulación, empezando el año escolar y los que se fueron agregando a lo largo del año lectivo, aspecto que disminuyó el ritmo de trabajo de las profesoras, ya que, las maestras los fueron integrando a las actividades a medida que fueron llegando a cada institución educativa, sin importar que tuvieran que devolverse sobre los logros ya alcanzados por los otros niños.

Con relación al trabajo de las docentes, se puede decir que la ejecución de las actividades, al inicio de la implementación, se llevó más tiempo que el estipulado por la investigadora, ya que no lograron integrarlas a la rutina de aula como se habría propuesto durante la fase de acción-colaboración, sino como un contenido temático más; después del trabajo colectivo realizado con las planeaciones de las unidades temáticas y los modelados realizados por la investigadora, cambió totalmente la dinámica de trabajo, lo que permitió utilizar las actividades de conciencia fonológica como mediaciones en el desarrollo de los contenidos curriculares de curso.

\section{Referencias}

Acosta, V., Moreno, A. y Axpe, M. (2011). Intervención sobre la conciencia fonológica en sujetos con trastorno específico del lenguaje en contextos inclusivos: 
posibilidades y limitaciones, Bordon, 63(3), 9-22. Retrieved from http://dialnet.unirioja.es/descarga/articulo/3712007.pdf

Andrade, L., Peña, K., Renowitzky, N. y Rincón, M, (2014). Efectos de un programa para la estimulación de las habilidades de conciencia fonológica en niños de kínder y Transición (tesis de Maestría inédita). Universidad del Norte, Barranquilla.

Arancibia, B., Bizama, M. y Sáez K. (2014). Aplicación de un programa de estimulación de la conciencia fonológica en preescolares de nivel transición 2 y alumnos de primer año básico pertenecientes a escuelas vulnerables de la provincia de Concepción, Chile. Revista Signos, 45(80), 236-256. Retrieved from https://www.redalyc.org/articulo.oa?id=157025024001

Auseche, P. (2009). Propuesta de formación docente para la transformación de la enseñanza del lenguaje escrito en grados iniciales. Universidad del Valle, Cali.

Caldera, R., Escalante, D. y Terán, M. (2010). Práctica Pedagógica de la lectura y formación docente. Revista de Pedagogía, 31(88). 15-37. Retrieved from http://www.scielo.org.ve/pdf/p/v31n88/art02.pdf

Defior, S. (2008). ¿Cómo facilitar el aprendizaje inicial de la lectoescritura? Papel de las habilidades fonológicas. Infancia y Aprendizaje, 31(3), 333-345. Retrieved from

https://www.researchgate.net/publication/233565490_Como_facilitar_el_aprend izaje_inicial_de_la_lectoescritura_Papel_de_las_habilidades_fonologicas_How does_facilitate_initial_literacy_learning_Phonological_abilities_role

Defior, S. y Serrano, F. (2011). Procesos fonológicos explícitos e implícitos, lectura y dislexia. Revista Neuropsicología, Neuropsiquiatría y Neurociencias, 11(1), 7994. Retrieved from https://dialnet.unirioja.es/descarga/articulo/3640858.pdf

Flórez, R., Restrepo, M., y Schwanenflugel, P. (2009). Promoción del alfabetismo inicial y prevención de las dificultades en la lectura: una experiencia pedagógica en el aula de preescolar. Avances en Psicología Latinoamericana, 27(1), 79-96. Retrieved from www.redalyc.org/pdf/799/79911627006.pdf

Flórez, R. (2009). Alfabetismo Emergente. Universidad Nacional de Colombia, Bogotá.

Fumagalli, J. y Jaichenco, V. (2010). Rendimiento diferencial en el reconocimiento de unidades fonológicas en dos grupos de niños prelectores de diferente proveniencia socioeconómica. En memorias $V$ congreso Marplatense de Psicología. Mar de Plata, Argentina, 369-371. Retrieved from https://es.scribd.com/doc/45172739/MEMORIAS-II-Congreso-Internacional-deInvestigacion-y-Practica-Profesional-en-Psicologia-XVII-Jornadas-deInvestigacion-Sexto-Encuentro-de-Investigado

Garay, E. (2012). Conocimiento de desarrollo fonológico y dificultades fonológicas en docentes del nivel inicial y primaria de colegios de fe y alegría Lima Este. Pontifica Universidad Católica del Perú, Lima.

Ghiglion, M., Arán, V. y Apaz, A. (2011). Programa de intervención, para fortalecer funciones cognitivas y lingüísticas, adaptado al currículo escolar en niños en riesgo por pobreza. Interdisciplinaria, revista de Psicología y Ciencias afines. 28 (1), 17-36. Retrieved from https://www.redalyc.org/articulo.oa?id=18022327002 
González, R., López, S., Vilar, J. y Rodríguez, A. (2013). Estudio de los predictores de la lectura. Revista de Investigación en Educación, 11 (2), 98-110. Retrieved from https://dialnet.unirioja.es/descarga/articulo/4734781.pdf

González, M., Martín, I., Delgado, M. (2011). Intervención temprana de la lectoescritura en sujetos con dificultades de aprendizaje. Revista Latinoamericana de Psicología, 43, (1, 2), 35-44. Retrieved from https://www.redalyc.org/articulo.oa?id=80520078004

Maldonado, G., y Guerrero, Z. (2009). La Mochila Mágica. Programa de Promoción de Lectura para la Educación Inicial. Acción Pedagógica, 17(1), 96-105. Retrieved from https://www.saber.ula.ve/bitstream/123456789/27486/1/articulo10.pdf

Martins, N., y Aparecida, C. (2009). Intervenção em habilidades metafonológicas em estudantes do ensino fundamental e desenvolvimento de leitura. Psicologia em Revista, 15(03), 140-158. Retrieved from http://pepsic.bvsalud.org/scielo.php?script=sci_arttext\&pid=S1677$\underline{11682009000300009}$

Mejía, L. y Eslava, J. (2008). Conciencia fonológica y aprendizaje lector. En L. Mejía y J. Eslava, Acta de Neurología (24): S55-S63. Retrieved from https://www.acnweb.org/acta/2008_24_S2_55.pdf

Ministerio de Educación Nacional (1994). General de Educación, Ley 115. Colombia: MEN

Porta, M. (2012). Un programa de intervención pedagógica en conciencia fonológica: efectos sobre el aprendizaje inicial de la lectura. Revista de orientación educativa 26(50), 93-111. Retrieved from https://dialnet.unirioja.es/servlet/articulo?codigo $=4554590$

Rodríguez, M., Cruz, V. y Portilla, De. (2010). Programa de Desarrollo de Habilidades Fonológicas y acceso inicial a la lectoescritura en alumnos y alumnas de 5 años del Nivel Inicial. Retrieved from https://es.scribd.com/document/327754231/58036990-PROGRAMACONCIENCIA-FONOLOGICA-pdf

Velarde, E., Canales, R., Meléndez, M., y Lingán, S. (2011). Programa de estimulación de las habilidades prelectores en niños y niñas de educación inicial de la provincia. Constitucional del Callao, Perú. Investigación Educativa, 15(27), 5373. Retrieved from http://sisbib.unmsm.edu.pe/bibvirtual/publicaciones/inv_educativa/2011_n27/a0 4v15n27.pdf

Viecco, S. y Rangel, K (2012). Experticia en el diseño y ejecución de actividades para la estimulación de la conciencia fonológica en los niveles de prejardín y jardín. (Tesis de Maestría no publicada). Universidad del Norte, Barranquilla.

Villagrán, M., Marchena, E., y Navarro, J. (2011). Niveles de dificultad de la conciencia fonológica y aprendizaje lector. Revista de Logopedia, Foniatría y Audiología, 31 (2), 96-105. Retrieved from http://www.elsevier.es/es-revista-revistalogopedia-foniatria-audiologia-309-articulo-niveles-dificultad-concienciafonologica-aprendizaje-S0214460311701772 
Fecha de recepción: 21/11/2017

Fecha de revisión: 07/05/2018

Fecha de aceptación: 26/07/2018 